\title{
Primary hydatid cyst of the stomach: a rare case report and review of the literature
}

\begin{abstract}
Hydatid cyst is an infestation disease of the humans that caused by Echinococcus Granulosus and can involve all organs and tissues that produce cyst everywhere in body. The most common organ of body for development of a hydatid cyst is the liver $(75 \%)$ and the lung $(15 \%)$. We present a primary stomach hydatid cyst resected via laparotomy with the affected stomach wall. As far as we know, this is the first report of a primary stomach hydatid disease in the case presentation: A 64year-old woman presented with a palpable mass on the epigastric and left upper quadrant of the abdomen. She had an 8month history of intermittent abdominal pain attacks, discomfort, weight loss, anemia and early satiety. Abdominal examination revealed a palpable, immobile mass at the epigastrium and left hypochondriac region. Laboratory findings were unremarkable. Abdominal U\&S and computed tomography (CT) scans revealed a heterogeneous mass originated from the stomach wall, spleen, pancreas or liver measuring $15 \times 8 \mathrm{~cm}$. No pathological appearance was detected in the chest x-ray. Upper GI endoscopy was also unrevealing during diagnostic laparotomy. The pancreas, liver and spleen were intact, but we found a huge cystic mass located at the anterior surface of body of stomach. The mass was taken out from inside of the abdomen. Stomach defect closed the pathology report revealed a hydatid cyst.
\end{abstract}

Volume 3 Issue 6 - 2017

\author{
Manoucheher Aghajanzadeh,' Mehdi \\ Karimian, ${ }^{2}$ Bahareh Malekzadeh, ${ }^{2}$ Zahra \\ Sadat Segatoleslami, ${ }^{3}$ Syyed Mostafa Zia \\ Ziabari, ${ }^{4}$ Hossein Torabi ${ }^{4}$ \\ 'Department of Thoracic and General Surgery, Guilan University \\ of Medical Sciences, Iran \\ ${ }^{2}$ Dpartement of General Surgery, Shahrekord University of \\ Medical Sciences, Iran \\ ${ }^{3}$ Shahid Beheshti University Medical Sciences, Iran \\ ${ }^{4}$ Resident of General Surgery, Guilan University of Medical \\ Sciences, Razi Hospital, Rasht, Iran
}

\begin{abstract}
Correspondence: Syyed Mostafa Zia Ziabari, Resident of General Surgery, Guilan University of Medical Sciences, Razi Hospital, Rasht, Iran, Email Smzziabari@yahoo.com
\end{abstract}

Received: October 17, 2017 | Published: November 28, 2017

Keywords: hydatid cyst, echinococcus granulosus, stomach hydatidosis, computed tomography, liver

Abbreviations: CT, computed tomography; GIST, gastrointestinal stromal tumor; ELISA, enzyme-linked immunosorbent assay; IHAT, indirect hemagglutination test; US, ultrasonography

\section{Introduction}

Hydatid disease is endemic in most parts of Iran and is hyper endemic in some areas. ${ }^{1-3}$ Hydatidosis is responsible for approximately $1 \%$ of admissions to surgical wards in Iran. ${ }^{1,4,5}$ The endemic areas, include the Mediterranean region, Africa, South America, Australia, Middle East and India. ${ }^{2,4,6}$ Hydatid cysts are mainly located in liver or lungs $(78 \%)$, But Liver is the most common involved organ. ${ }^{1-4}$ Hydatid cysts can be found in almost any organ of the body but the most common sites are liver (50\%-77\%), lung (15\%-47\%), spleen $(0.5 \%-8 \%)$, and kidney $(2 \%-4 \%))^{1,5,7,8}$ The rest of the sites include muscle, peritoneum, bone, pancreas, heart, and brain. ${ }^{2-4}$ Patients who have an extrahepatic hydatid cyst present mostly with abdominal pain and discomfort. ${ }^{7,8}$ Diagnosis can be challenging. ${ }^{2-8}$ We present here an unusual case of primary gastric hydatid cyst and a review of the literature makes this a rare case and as far as we know, this is the first report of a primary stomach hydatid disease in the literature.

\section{Case presentation}

A 64-year-old woman presented with an 8-months history of intermittent abdominal pain attacks, vomiting, abdominal discomfort, weight loss, anemia and early satiety. Abdominal examination revealed a huge palpable, immobile mass at the left hypochondriac, epigastric and left upper quadrant region with extension to the right upper quadrant and liver. Head, neck, Chest lower abdomen and extremity exams was normal. The patient's past medical history and family history were unrevealing. Laboratory findings (liver function, renal tests, PT, PTT, INR) were unremarkable $\mathrm{WBC}=12000, \mathrm{HB}=10$, albumin $=2.5 \mathrm{~g} / \mathrm{Li}$. Hydatid serology (IHA) and immunologic (anti hydatid antibody) tests were negative. Abdominal ultrasonography (U\&S) revealed a homogeneous cystic mass originated from the spleen, pancreas, liver or stomach wall, mesearing $15 \times 8 \mathrm{~cm}$. the origin of cystic mass was not identified with (U\&S) definitely abdominal computed tomography scans revealed a heterogeneous mass located between spleen, pancreas, stomach and left lobe of liver mesearing 15 $\mathrm{x} 8 \mathrm{~cm}$ (Figures 1,2). The origin of cystic mass was not also identified with (CT). In the chest $\mathrm{x}$-ray, no pathological finding was detected. Upper GI endoscopy was also normal. A preliminary diagnosis of hydatid cyst of liver and spleen and pseudo cyst and hydatid cyst of pancreas or a gastrointestinal stromal tumor (GIST) originating from the stomach was made. During diagnostic laparotomy, a huge totally cystic lesion between pancreas, spleen, stomach and liver, after walling of the location and around the cyst sailen and sponge, about $700 \mathrm{cc}$ fluid was aspirated from cyst (Figure 3), totally the cyst was collapsed without any solid component, cyst was opened and laminated layers was exposed (Figure 4). The laminated membrane was taken out of the abdomen (Figure 5). For finding the origin of cyst exploration was done and pancreas, spleen, and liver was intact and no connection was between cysts with these organs. But we found a $5 \mathrm{~cm}$ connection located at the body of the stomach between mucosa and muscular layers. During dissection and resection of cyst layer a perforation was presented in the stomach wall and repaired in two layers (Figure 6). The postoperative period was uneventful and the patient was discharged on day six.

The pathology report revealed cyst on the serosa aspect of the stomach. These histological and morphological findings supported a diagnosis of a hydatid cyst. Postoperative, A three-cycle Albendazole treatment was ordered to the patient. (400mg Albendazole twice a day for 28days unstopped for 14days). No recurrence was noted during the 6-month follow-up period. 


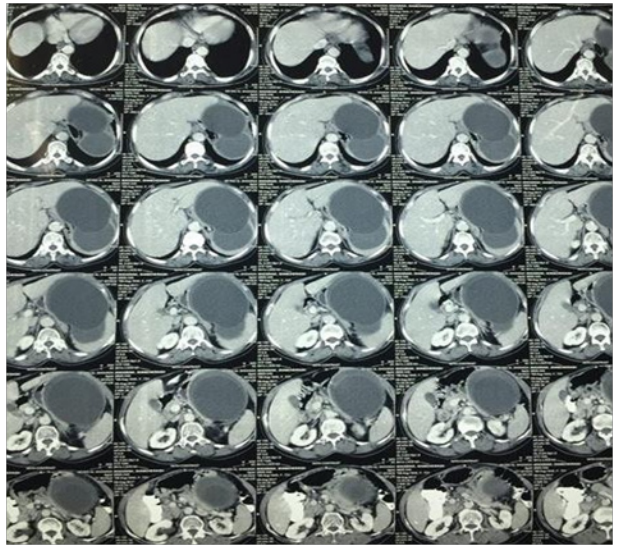

Figure I CT scan of abdomen with IV contrast show two huge cysts between spleen, stomach, liver and pancreas.

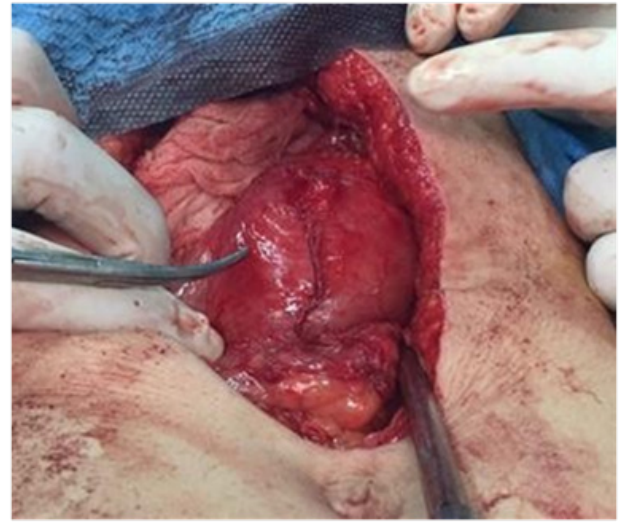

Figure 2 Cyst and stomach.

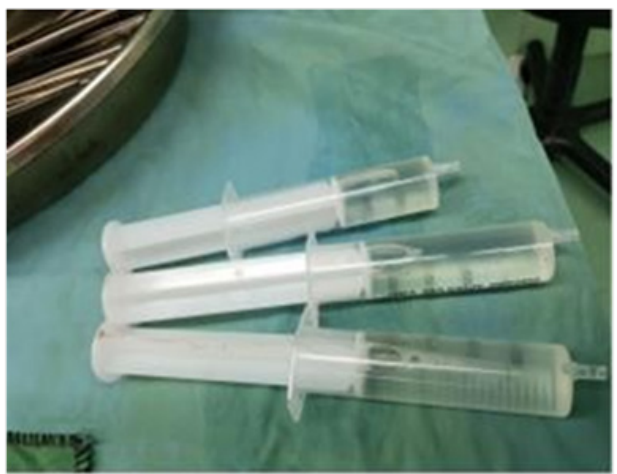

Figure 3 Aspiration fluid of cyst.

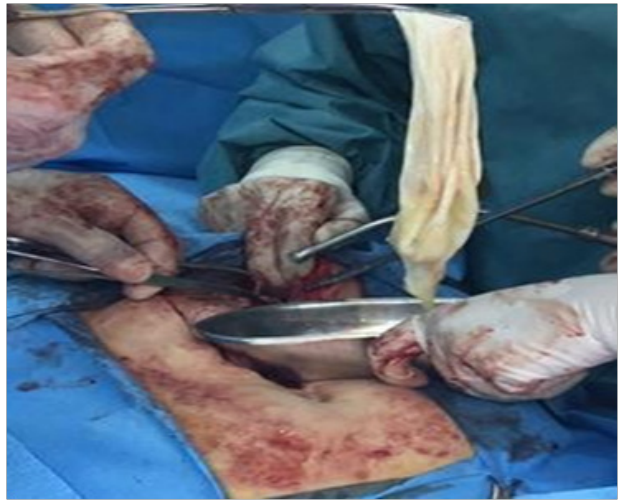

Figure 4 Laminated membrane of cyst.

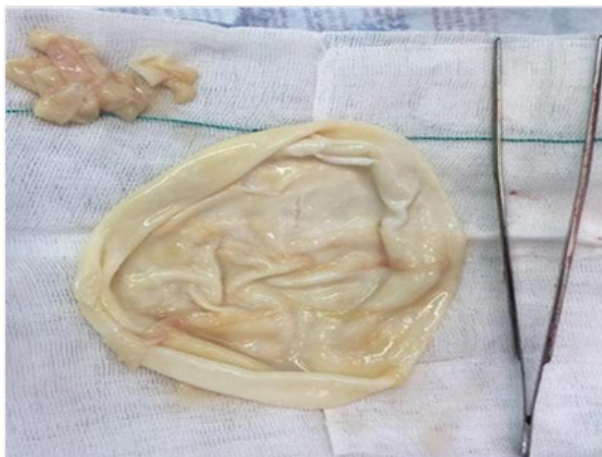

Figure 5 Laminated membrane after removal.

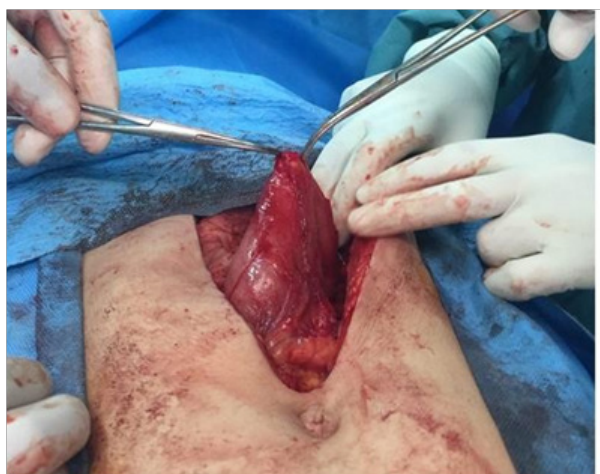

Figure 6 The orifice of cyst on the stomach.

\section{Discussion}

Hydatid disease, caused by the Echinococcus granulosus is an endemic disease especially in regions like Eurasia and South American countries. ${ }^{1-3}$ The stomach is an unusual site for a hydatid cyst, and there are a few primary peritoneal hydatid cyst case reports in the literature. ${ }^{1-3,7,8}$ The mechanism of infestation is not clear. ${ }^{2,3}$ For stomach, retropritoan and intestinal mesenteric hydatid cysts, lymphatic or systemic circulation dissemination has been implicated as a possible route for organs involvement, as in our case, we think lymphatic or systemic circulation might also be the mechanism in our case. Extrahepatic hydatid cyst usually remains asymptomatic for years. ${ }^{4-6,9}$ Patients mostly present after the cyst becomes large enough to palpate or to cause non-specific symptoms as abdominal discomfort vomiting as our case..$^{1,2,7-9}$ The combination of clinical, laboratory, radiological findings upper and lower GI endoscopy help for a preliminary diagnosis. Among all imaging modalities, ultrasonography is superior to both MRI and CT in visualization and evaluation of the morphology of liver cysts and hydatid cyst as in our case we didn't use MRI. We used U\&S in first step. ${ }^{10}$ In sensitivity rates of ultrasonography for diagnosis of hydatid cyst are $(88 \%$ to $98 \%)$ and specificity are (93\% to $100 \%) .{ }^{10}$ Abdominal cystic lesions as pesudocyst of pancreas, spleen cysts, mesenteric cysts, ovarian cysts and lymphangioma, must be considered in the differential diagnosis. ${ }^{8}$ In our practice, the workup of a patient with an abdominal mass, we start with an ultrasonography and for definitive anatomical localisation. We use CT scan. If the CT scan reported a non-cystic mass originating from the stomach wall, we recommended upper and lower GI endoscopy for malignant lesion especially as our patient with weight loss and anemia .Commonly used serological methods in the diagnosis of hydatid disease are the enzyme-linked immunosorbent assay (ELISA), the indirect hemagglutination test (IHA), the latex agglutination test and immunoblots. ${ }^{7-9}$ But we routinely didn't use 
serologic and immunologic tests for diagnosis of hydatid cysts. Because, the usage of these tests still remain controversial due to inadequate sensitivity and specificity rates. ${ }^{2}$ Nevertheless, the usage of these tests still remains controversial due to inadequate sensitivity and specificity rates. A wide range of sensitivity rates (50\% to $100 \%)$ and the specificity rate is also reported to be $83 \%$ to $88 \%{ }^{2,7}$ In our case, anti cystode antibody (ELISA) was negative. The gold standard treatment for hydatid cyst is complete surgical excision though according to the site of origin, without any spilage of hydatid material, In our case one of diagnosis was the hydatid cyst of liver or spleen, during anesthesia induction $100 \mathrm{mg}$ hydrocortisone was injected and all of the anaphylaxis events were ready .laparotomy was performed, after expose of cystic mass all side of cyst was walling offend partial, after aspiration (Figure 3). Subtotal cystectomy was performed to avoid adjacent organ injuries. Mebendazole or Albendazole is given to the patient adjuvantly to prevent recurrence. ${ }^{2}$ In our case, we preferred Albendazole treatment, ${ }^{4-6}$ and there was no recurrence in the 6-month follow-up period. As far as our knowledge, this is the first case report of a primary stomach hydatid cyst was resected via laparotomy.

\section{Conclusion}

Hydatid disease is a significant public health problem in under developed and developing countries affecting mostly the liver and lungs. Primary stomach hydatid cyst is a very rare clinical entity, which should be kept in mind for patients with an intra-abdominal mass. It is an extremely unusual condition and should be included in the differential diagnosis of stomach, splenic and pancreatic mass or cystic lesions especially in endemic areas.

\section{Acknowledgements}

None.

\section{Conflicts of interest}

The authors declared that there are no conflicts of interest.

\section{References}

1. Ahmed Z, Chhabra S, Massey A, et al. Primary hydatid cyst of pancreas: Case report and review of literature. Int J Surg Case Rep. 2016;27:74-77.

2. Tarahomi M, Otaghvar HA, Ghavifekr NH, et al. Primary Hydatid Cyst of Umbilicus, Mimicking an Umbilical Hernia. Case Reports in Surgery. 2016;2016:Article ID 9682178.

3. Ertekin SC, Ozmen T. Primary Hydatid Cyst of the Small Intestine: A Rare Case Report and Brief Review of the Literature. Cureus. 2016;28:8(7).

4. Eamaeili Delshad MS, Aghajanzadeh M, Hossein Hemmati, et al. Complicated primary Hydatid Cyst in brachioradialis muscle: An extremely unusual site. Merit Res J Med Med Sci. 2016;4(2):76-78.

5. Aghajanzadeh M, Alavi CE, Asgary MR, et al. Primary Hydatid Cyst of the Rib: Present as a Chest Wall Mass. MOJ Surgery. 2016;3(2):00041.

6. Aghajanzadeh, Karimian M, Segatoleslami ZS, et al. Primary Isolated Hydatid Cyst in Trapezes Muscle: A Extremely Rare Site Manoucheher. Annals of Clinical Pathologyl. 2017;5(3):1111.

7. Pandya JS, Bhambare MR, Waghmare SB, et al. Primary hydatid cyst of peritoneum presented as abdominal lump: a rare presentation. Clin Case Rep. 2015;3(5):331-333.

8. Wani RA, Malik AA, Chowdri NA, et al. Primary extrahepatic abdominal hydatidosis. Int J Surg. 2005;3(2):125-127.

9. Khare DK, Bansal R, Chaturvedi J, et al. Primary peritoneal echinococcosis masquerading as an ovarian cyst. Indian J Surg. 2006;68(3):181-183.

10. Wuestenberg J, Gruener B, Oeztuerk S, et al. Diagnostics in cystic echinococcosis: serology versus ultrasonography. Turk J Gastroenterol. 2014;25(4):398-404. 